\title{
Rényi entropy of a line in two-dimensional Ising models
}

\author{
J.-M. Stéphan, ${ }^{1}$ G. Misguich, ${ }^{1}$ and V. Pasquier ${ }^{1}$ \\ ${ }^{1}$ Institut de Physique Théorique, CEA, IPhT, CNRS, URA 2306, F-91191 Gif-sur-Yvette, France.
}

('Dated: June 9th, 2010)

\begin{abstract}
We consider the two-dimensional (2d) Ising model on a infinitely long cylinder and study the probabilities $p_{i}$ to observe a given spin configuration $i$ along a circular section of the cylinder. These probabilities also occur as eigenvalues of reduced density matrices in some Rokhsar-Kivelson wavefunctions. We analyze the subleading constant to the Rényi entropy $R_{n}=1 /(1-n) \ln \left(\sum_{i} p_{i}^{n}\right)$ and discuss its scaling properties at the critical point. Studying three different microscopic realizations, we provide numerical evidence that it is universal and behaves in a step-like fashion as a function of $n$, with a discontinuity at the Shannon point $n=1$. As a consequence, a field theoretical argument based on the replica trick would fail to give the correct value at this point. We nevertheless compute it numerically with high precision. Two other values of the Rényi parameter are of special interest: $n=1 / 2$ and $n=\infty$ are related in a simple way to the Affleck-Ludwig boundary entropies associated to free and fixed boundary conditions respectively.
\end{abstract}

\section{INTRODUCTION}

The entanglement (or Von Neumann) entropy is in general a difficult quantity to compute in two-dimensional quantum lattice models. In Ref. 2 it was however shown that for a particular type of wave functions, of type dubbed "Rokhsar-Kivelson" (RK), and for particular geometries, the calculation simplifies considerably. A lattice model of statistical mechanics can be used to define a Rokhsar-Kivelson wave-function as follows:

$$
|\mathrm{RK}\rangle=\frac{1}{\sqrt{\mathcal{Z}}} \sum_{c} e^{-\frac{1}{2} E(c)}|c\rangle .
$$

where the sum runs over the classical configurations and $E(c)$ is the energy associated to $c$ (interactions are assumed to be short-ranged), and the normalization factor involves the classical partition function $\mathcal{Z}$. For such a state, it has been shown in Ref. 5 that the eigenvalues of the reduced density matrix of a semi-infinite cylinder (with a finite circumference $L$, see Fig. 11) are simply the classical probabilities $p_{i}$ to observe a given configuration $i$ at the boundary between $A$ and $B$. In turn, these probabilities can be obtained from the dominant eigenvector of the transfer matrix of the classical model. So, the complete entanglement spectrum is encoded in the dominant eigenvector of the classical transfer matrix.23 In this work, we concentrate on the situation where the classical model is a two-dimensional Ising model. Each probability $p_{i}$ is therefore associated to a given configuration $i$ of the spins along the "ring" of length $L$ which separates the regions $A$ and $B$ (Fig. 1). Specifically, we are interested in the behavior of the Rényi entropies

$$
R_{n>0}=\frac{\ln \left(Z_{n}\right)}{1-n} \quad Z_{n}=\sum_{i} p_{i}^{n}
$$

including its limit

$$
\lim _{n \rightarrow 1} R_{n}=-\sum_{i} p_{i} \ln \left(p_{i}\right),
$$

which is the Shannon entropy (or Von Neumann in the quantum/RK point of view $)$.

As discussed in previous studies, $50 R_{n}(T, L)$ scales linearly with perimeter $L$ of the cylinder, even at the critical temperature. However, the most interesting piece of information is the first subleading correction, $r_{n}(T)$. For a given temperature $T$, the later is defined through an expansion of $R_{n}(T, L)$ for large $L$ :

$$
R_{n}(T, L) \simeq a_{n}(T) L+r_{n}(T)+o(1)
$$

and is of order one.24 Contrary to the coefficient $a_{n}, r_{n}$ have been argued to be universal. In the case of Ising models, $r_{1}\left(T>T_{c}\right)=0$ in the high temperature phase and $r_{1}\left(T<T_{c}\right)=\ln (2)$ in the low temperature phase. At the critical point, the previous numerical calculations (up to $L=36$ ) lead to $r_{1}\left(T=T_{c}\right) \simeq 0.2544 .25$ The numerical results presented in Sec. II significantly increase the precision on this number: $r_{1}\left(T_{c}\right)=0.2543925(5)$. Furthermore, we confirm its universal character by checking the agreement between three microscopically different realizations of the $2 \mathrm{~d}$ critical Ising models: on the square and triangular lattice, and using the Ising chain in transverse field (ICTF). At present, we are not aware of any field theory method which is able to compute this number.

In Sec. III we analyze the finite-size scaling of $r_{1}(\mu, L)$ in the vicinity of the critical point, using numerical (but exact) calculations for the ICTF. There, the parameter $\mu$ measures the ratio of the spin-spin interaction over the strength of the external magnetic field and plays the role of the temperature in the classical Ising model. Away from $\mu=\mu_{c}$, we conclude that $r_{1}(\mu, L)$ only depends on $L(\mu-1)$ in the critical regime, which is consistent with a correlation length diverging as $1 /(\mu-1)$ close to the critical point (located at $\mu_{c}=1$ ). In particular, we confirm the step-like shape of $r_{1}(\mu, L=\infty)$.

In Sec. IV we analyze the finite-size scaling of $r_{n}(\mu=$ $\left.\mu_{c}=1, L\right)$ in the vicinity of $n=1 / 2$ and $n=1$, again with the ICTF (up to $L=44$ sites). The case $n=1 / 2$ turns out to be exactly solvable (Sec. 6) and related to the "ground-state degeneracy" for a critical Ising model 


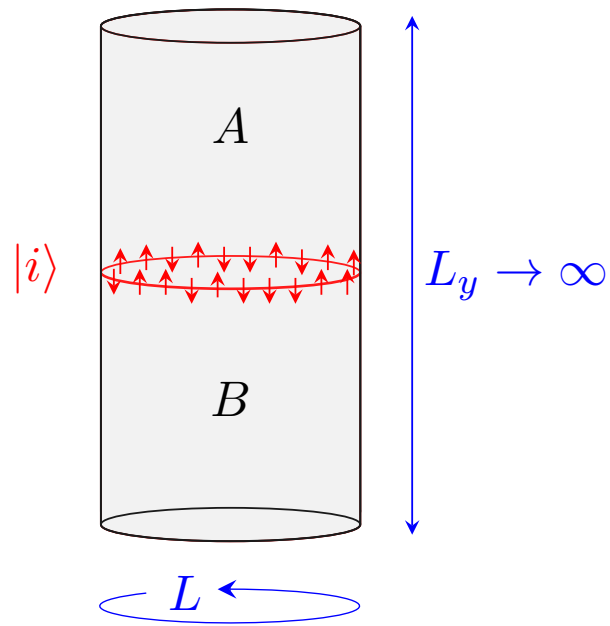

FIG. 1. (Color online) Cylinder geometry with $L_{y} \gg L$. A probability $p_{i}$ is associated to each spin configuration $i$ of the boundary (red circle) between $A$ and $B$.

with free bqundary conditions, as discussed by Affleck and Ludwig. In the vicinity of $n=1$ the numerical data strongly suggests a step-like shape of $r_{n}(\mu=1, L=\infty)$ as a function of the Rényi parameter $n: r_{n}(\mu=1, L=$ $\infty)=0$ for $n<1$ and $r_{n}(\mu=1, L=\infty)=\ln 2$ for $n>1$. This result has some important consequence regarding possible field theory approaches. In particular, a singularity at $n=1$ would invalidate any attempt to compute $r_{1}$ from an analytical continuation to $n=1$ of the $n \in \mathbb{N}^{*}$ result (replica trick).

\section{SHANNON ENTROPY AT THE CRITICAL POINT}

\section{A. Square and triangular lattices}

We compute the Shanon entropy $R_{1}$ using the transfer matrix $\mathcal{T}$ of the ferromagnetic Ising model. We numerically diagonalize $\mathcal{T}$ (in the full space of dimension $2^{L}$ ), on the square and on the triangular lattices 26 for sizes up to $L=14$ and denote by $|L\rangle$ and $|E\rangle$ the left- and rightdominant eigenvectors of $\mathcal{T}$ (corresponding to the eigenvalue with the largest modulus). Then, the probability $p_{i}$ of a configuration $i$ is given by:

$$
p_{i}=\frac{\langle L \mid i\rangle\langle i \mid R\rangle}{\langle L \mid R\rangle}
$$

in the limit of a infinitely long cylinder $L_{y} \gg L$.

The results for $R_{1}\left(T_{c}\right)$, obtained by summing over the $2^{L}$ configurations, are shown in Fig. 2. The linear behavior, $R_{1}\left(T_{c}\right) \sim L$ is apparent, as well as the fact that the data for the two lattices extrapolate to the same value $\simeq 0.254$ at $L=0$. Although the systems are relatively small, it shows that $r_{1}\left(T_{c}\right) \simeq 0.254$ does not depend on the microscopic lattice geometry, and is therefore very likely to be universal.

\section{B. Ising chain in transverse field}

As a third microscopic realization of the Ising $2 \mathrm{~d}$ universality class, we study the ICTF:

$$
\mathcal{H}=-\mu \sum_{j=0}^{L-1} \sigma_{j}^{x} \sigma_{j+1}^{x}-\sum_{j=0}^{L-1} \sigma_{j}^{z} .
$$

This Hamiltonian proportional to the logarithm of the transfer matrix of an anisotropic Ising model on the square lattice, with couplings along the $y$ direction ("time") which are much stronger than in the $x$ direction ("space") .

This Hamiltonian is transformed into a free fermion problem using the standard Jordan-Wigner transformation. The later free fermion problem is then diagonalized using a Bogoliubov transformation. The ground-state of $\mathcal{H}$ is then described as the vacuum of the Bogoliubov fermions. The critical point is located at $\mu=1$. For $\mu>1$ the system is in the ordered phase, with spontaneously broken $\mathbb{Z}_{2}$ symmetry $\left(\left\langle\sigma^{x}\right\rangle \neq 0\right)$, and for $\mu<1$ the system is in the disordered (paramagnetic) phase.

It turns out that the ground-state $|G\rangle$ of the chain is simpler to express in $\sigma^{z}$ basis. For an Ising spin configuration $|i\rangle$ labeled by the variables $\sigma_{i}^{z}= \pm 1$, the probability at $\mu=1$ is :

$$
p_{i}=|\langle i \mid G\rangle|^{2}=p\left(\sigma_{0}^{z}, \cdots, \sigma_{L-1}^{z}\right)=\operatorname{det} M
$$

where $M$ is an $L \times L$ matrix defined by:

$$
M_{j \ell}=\frac{1}{2} \delta_{j \ell}+\frac{(-1)^{j-\ell} \sigma_{j}^{z}}{2 L \sin \left[\pi\left(j-\ell+\frac{1}{2}\right) / L\right]}
$$

This result is derived in Appendix. A, where the noncritical case $\mu \neq 1$ is also considered. However, going back to the initial $2 \mathrm{~d}$ classical model, the actual spin directions are measured by $\sigma_{i}^{x}$. So, we first compute an entropy $R_{n}^{(z)}$ corresponding to probabilities of $z$-axis configurations, and then use the Kramers-Wannier duality transformation 12 to obtain the desired $R_{n}=R_{n}^{(x)}$ :

$$
R_{n}^{(x)}(\mu)=R_{n}^{(z)}(1 / \mu)+\ln 2 .
$$

The calculation of $R_{n}^{z}$ amounts to compute $2^{L}$ probabilities, each of which is obtained as a determinant of size $L \times L$. Using the translation invariance and the reflection symmetry of the chain, the number of probabilities to compute can be reduced to $\sim 2^{L} /(2 L)$.27 To do so we generate one representative for each orbit of spin configurations (under the action of the lattice symmetries) using the "bracelets" enumeration algorithm of Ref. 13. For the largest size, $L=44$, computing all the probabilities $\left(2^{L}=1.7 \times 10^{13}\right)$ required about one thousand hours of CPU time on a parallel machine. 


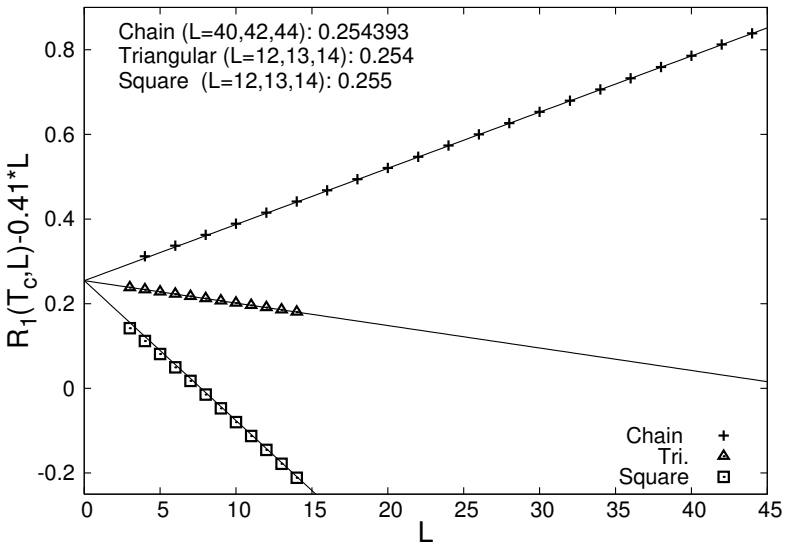

FIG. 2. Shannon entropy $R_{1}\left(T_{c}, L\right)$ of the Ising models at the critical point, plotted as a function of $L$ (a linear term, $-0.41 L$, has been subtracted for clarity). Data for the square and triangular lattices and for the Ising chain in transverse field are compared. The data are well reproduced by $R_{1}(L) \simeq$ $a L+r_{1}+b / L$ and the subleading constant $r_{1}$ is evaluated using the three largest sizes. Each line represents the leading term and the constant, $a L+r_{1}$. The subleading term appears to be the same $r_{1} \simeq 0.254$ for the three microscopic models.

The data for the Shannon entropy $R_{1}$ are plotted in Fig. 2 and given in Table 1 . They significantly extend the results published in Ref. 5 . The columns $r_{1}^{(1)}, r_{1}^{(4)}$ and $r_{1}^{(5)}$ correspond to three different ways to extract the subleading constant from $R_{1}(\mu=1, L)$, with three different types of fits (details in the table caption). In all cases the result rapidly converges and, using the largest size ( $L=44$ spins) we estimate that $r_{1}=0.2543925(5)$ at $L=\infty$.

\section{III. $\mu$ AWAY FROM THE CRITICAL POINT}

In this section, we investigate the behavior of $r_{1}$ in the vicinity of the critical point, by considering the Ising chain in transverse field away from $\mu=1$. The results are summarized in Fig. 3 .

In this plot, $r_{1}(\mu)$ is extracted from $R_{1}(L, \mu)$ using a fit to $a_{1}(\mu) L+r_{1}(\mu)+b_{1}(\mu) / L$ with three consecutive values of $L$. For the size we have studied (here $L \leq 38$ ), there is still some visible finite-size effects. In particular, the marked oscillations in the vicinity of $\mu=1$ are not converged to the $L=\infty$ limit. In fact, it is reasonable to expect the curves to gradually approach a step-like function as $L$ increases: $r_{1}=0$ for $\mu<1$ and $r_{1}=\ln (2)$ for $\mu>1$.

This scenario, anticipated in Ref. 5 , is corroborated by the scaling shown in the inset of Fig. 3. When plotted as a function of $(\mu-1) L$, the data for different system sizes and different values of $\mu$ collapse onto a single - and very likely universal - curve. This can be understood from the fact that the correlation length $\xi$ of the Ising model

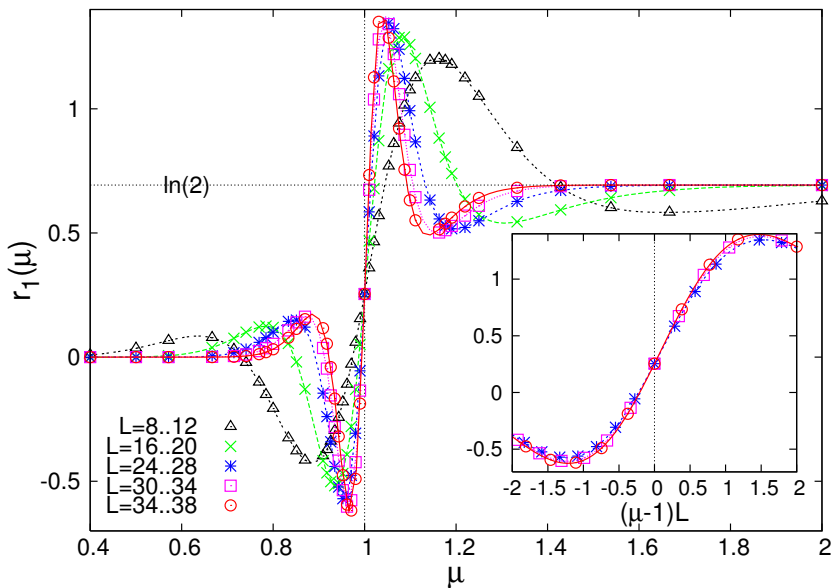

FIG. 3. Subleading constant $r_{1}$ of the Shannon entropy of the Ising chain as a function of $\mu$. The critical point corresponds to $\mu=1$. Inset: $r_{1}(\mu)$ for different system sizes, plotted as a function of $(\mu-1) L$.

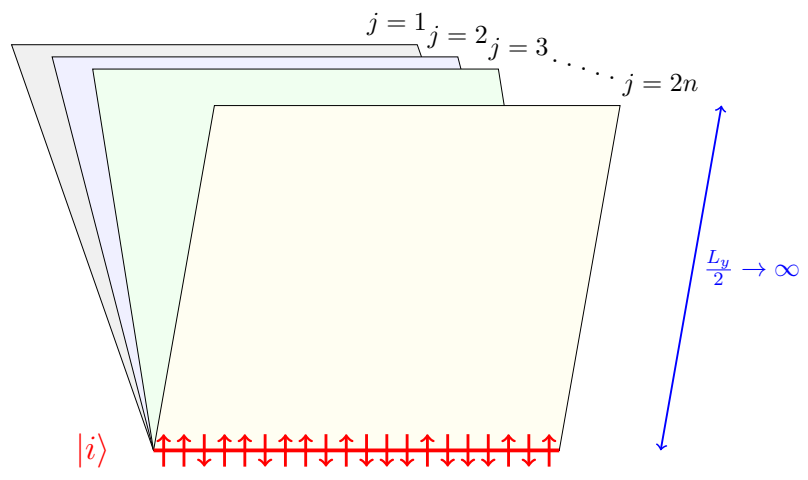

FIG. 4. $2 n$ Ising models glued together at their boundary ("Ising book"). In our case, each "page" has periodic boundary conditions along the horizontal axis and is semi infinite in the vertical direction. Fig. 1 corresponds to 2 pages $(n=1)$.

diverges as $1 /|\mu-1|$ at the transition, and if one assumes that $r_{1}$ is a function of $L / \xi(\mu)$ in the critical region. If correct, it immediately implies that $r_{1}(\mu)$ is a step-like function in the thermodynamic limit.

\section{RÉNYI ENTROPY AWAY FROM $n=1$}

We now consider the effect of changing the Rényi parameter $n$. When $2 n$ is an integer, $R_{n}$ has an interpretation in terms of the free energy of $k=2 n$ semi-infinite Ising models which are "glued" together at their boundary (see Fig. 价).

Using the transfer matrix point of view, it is simple to see that $p_{i}^{k / 2}$ is (proportional to) the probability to observe the spin configuration $i$ on a circle along which $k$ Ising models (defined on semi-infinite cylinders) are forced to coincide. This was used in Refs. 6 and 7 in 


\begin{tabular}{|c|c|c|cccc|}
\hline & & & & \\
& & $a_{1}$ & $r_{1}^{(1)}$ & $r_{1}^{(4)}$ & $r_{1}^{(5)}$ \\
\hline 16 & 7.02789845748593 & 0.4232735600 & 0.2544012149 & 0.2543924985 & 0.2543925471 \\
18 & 7.87432026832476 & 0.4232735603 & 0.2543983072 & 0.2543925177 & 0.2543925302 \\
20 & 8.72076710746883 & 0.4232735604 & 0.2543965648 & 0.2543925180 & 0.2543925183 \\
22 & 9.56723215961776 & 0.4232735605 & 0.2543954570 & 0.2543925156 & 0.2543925130 \\
24 & 10.4137108773778 & 0.4232735605 & 0.2543947190 & 0.2543925136 & 0.2543925110 \\
26 & 11.2602001105626 & 0.4232735606 & 0.2543942083 & 0.2543925110 & 0.2543925072 \\
28 & 12.1066976079502 & 0.4232735605 & 0.2543938437 & 0.2543925139 & 0.2543925188 \\
30 & 12.9532017180203 & 0.4232735608 & 0.2543935763 & 0.2543925001 & 0.2543924741 \\
32 & 13.7997112017585 & 0.4232735600 & 0.2543933760 & 0.2543925306 & 0.2543925939 \\
34 & 14.6462251114521 & 0.4232735614 & 0.2543932227 & 0.2543924796 & 0.2543923635 \\
36 & 15.4927427098430 & 0.4232735597 & 0.2543931036 & 0.2543925326 & 0.2543926640 \\
38 & 16.3392634147881 & 0.4232735610 & 0.2543930095 & 0.2543925037 & 0.2543924262 \\
40 & 17.1857867605076 & 0.4232735606 & 0.2543929343 & 0.2543924999 & 0.2543924890 \\
42 & 18.0323123698967 & 0.4232735603 & 0.2543928734 & 0.2543925161 & 0.2543925658 \\
44 & 18.8788399343835 & 0.4232735602 & 0.2543928237 & 0.2543925300 & 0.2543925757 \\
\hline
\end{tabular}

TABLE I. (Color online) Shannon entropy $R_{1}(L, \mu=1)$ of the critical Ising chain in transverse field as a function of the system size $L$. The subleading constant $r_{1}$ is extracted using three different fits: $r_{1}^{(1)}$ is obtained by a fit to $R_{1}(L) \simeq a L+r_{1}+b L^{-1}$ using the three following system sizes: $L, L-2, L-4 . r_{1}^{(4)}$ is obtained by a fit to $R_{1}(L) \simeq a L+r_{1}+b L^{-1}+\cdots e L^{-4}$ using the six system sizes $L, L-2, \cdots, L-10 . r_{1}^{(5)}$ is obtained by a fit to $R_{1}(L) \simeq a L+r_{1}+b L^{-1}+\cdots f L^{-5}$ using the seven system sizes $L, L-2, \cdots, L-12 . a$ is the coefficient of the extensive (and non-universal) term, extracted from the seven-point fit above. From this analysis, our best estimate for $L=\infty$ is $r_{1}=0.2543925(5)$.

some field theory calculations, but it is also true at the microscopic level. The interpretation above does not apply when $2 n$ is not a positive integer, but $R_{n}(L, \mu)$ can still be computed numerically for any $n \geq 0$.

\section{A. Rényi parameter $n=2$ and above}

When $n$ goes to infinity, only the spin configuration with the largest probability contributes to $R_{n}$. For the ferromagnetic Ising models we consider (including the quantum chain in transverse field), this configuration is two-fold degenerate and corresponds to a fully polarized ferromagnetic state, $|\uparrow \uparrow \cdots \uparrow\rangle$ or $|\downarrow \downarrow \cdots \downarrow\rangle$. In other words, taking the limit $n \rightarrow \infty$ amounts to study a semiinfinite Ising model with ferromagnetic boundary conditions. The corresponding probability, $p_{\max }$, behaves as $-\ln \left(p_{\max }\right) \sim a L+\ln (2)$ at the critical point. The subleading constant, $\ln (2)$, is nothing but (twice) the " $g$ factor" associated to this conformally invariant boundary condition (more details in Sec. IVB). This implies for the Rényi entropies that the subleading constant $r_{n}\left(\mu_{c}\right)$ is $\ln (2)$ at $n=\infty$.

In fact, for $n \gtrsim 2$ the Fig. 5 shows that even relatively small systems give $r_{n}\left(\mu_{c}\right)$ very close to $\ln 2$. Table II is an analysis showing that $r_{2}(\mu=1) \simeq \ln 2$ with a great accuracy, of the order of $10^{-8}$. Since the convergence to $\ln 2$ is even faster when $n>2$, there is practically no doubt that $r_{n}\left(\mu_{c}\right)$ is exactly $\ln 2$ for $n \gtrsim 2.28$

As a consequence, an analytical continuation of this result to $n=1$ would erroneously give $r_{1}\left(\mu_{c}\right)=\ln 2$ (instead of 0.25439$)$. In particular, we note that the results of Ref. 17 (which use a replica technique) are in agreement with ours for $n>1$, but not at $n=1$.

$$
\text { B. } n=\frac{1}{2}
$$

The special value $n=\frac{1}{2}$ corresponds to the free energy of a single Ising model defined on a semi-infinite cylinder (keeping only part $A$ in Fig. 11), and can be treated exactly. Using the transfer matrix point of view, it is indeed simple to see that $\sqrt{p_{i}}$ is proportional to the probability to observe the spin configuration $i$ at the edge of a semi-infinite Ising model (contrary to $p_{i}$ which is the probability to observe $i$ in the bulk).

As far as the universal properties are concerned, we can study the ground state of the quantum Ising chain (Eq. (6) ) rather than the transfer matrix of the classical $2 \mathrm{~d}$ model. Denoting by $|G\rangle$ the ground-state of the chain, we have $\sqrt{p_{i}}=\langle G \mid i\rangle$ and the Rényi entropy $R_{\frac{1}{2}}$ can be written as:

$$
\begin{aligned}
R_{\frac{1}{2}}(L) & =2 \ln \left(\left\langle G\left|\sum_{\left\{\sigma_{i}^{x}= \pm 1\right\}}\right| \sigma_{1}^{x} \cdots \sigma_{L}^{x}\right\rangle\right) \\
& \left.=2 \ln \left(2^{L / 2}\langle G| \text { free }\right\rangle\right)
\end{aligned}
$$

where $\mid$ free $\rangle=\left|\left\{\sigma_{i}^{z}=1\right\}\right\rangle$ is the state where all the spins point in the $z$ direction. It turns out that the latter state is the vacuum of Jordan-Wigner Fermion and that the scalar product in Eq. (11) can be obtained as a particular case of Eqs. (78). At the critical point $(\mu=1)$, the result 


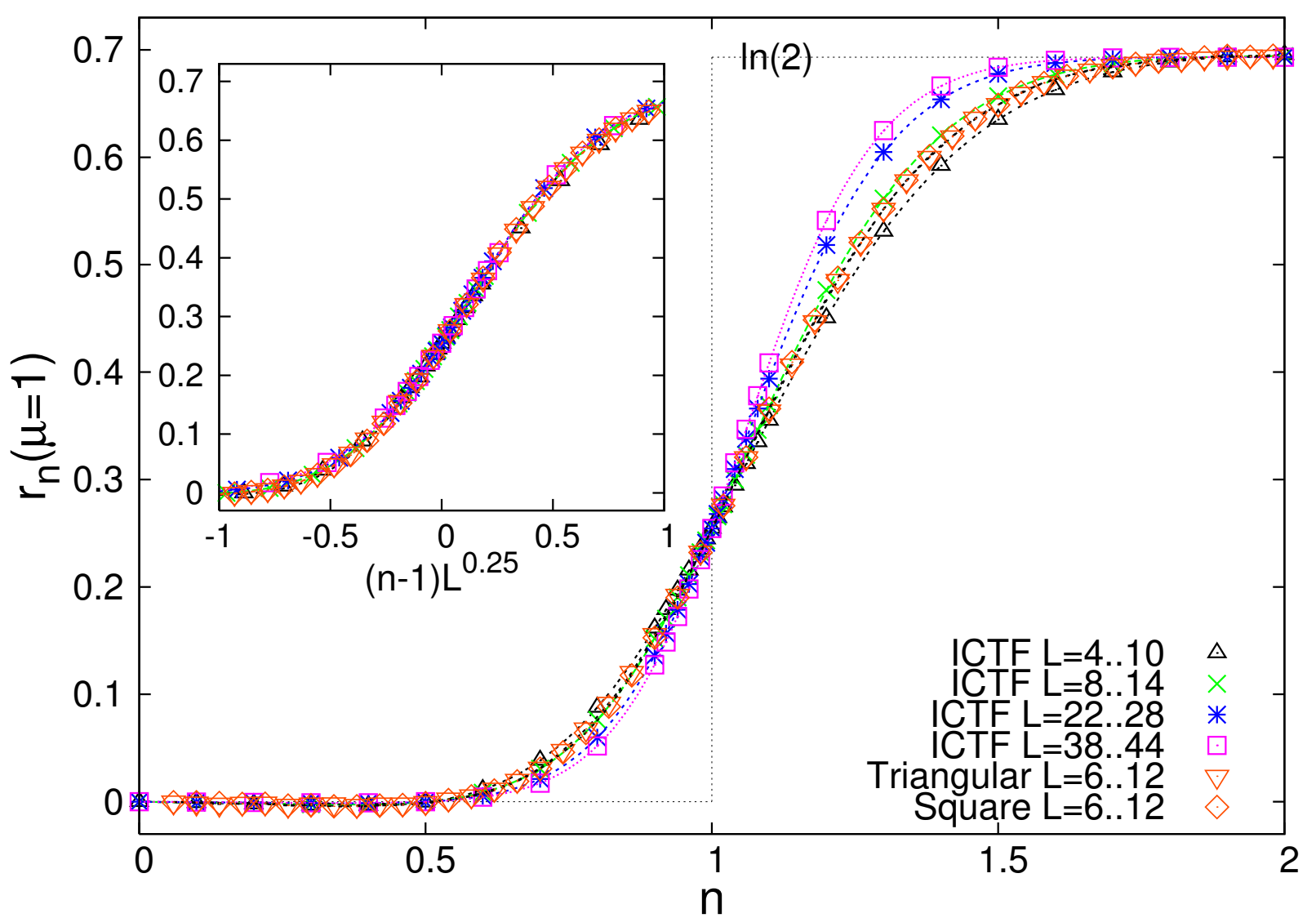

FIG. 5. (color online) Subleading constant $r_{n}$ of the Rényi entropy of the Ising chain (ICTF, at $\mu=1$ ) and classical Ising models (triangular and square lattice, at $T=T_{c}$ ) as a function of the Renyi parameter $n$. The (slow) convergence towards a step function can be observed. Inset : when plotted as a function of $(n-1) L^{0.25}$, the data collapse reasonably well onto a single curve. For each value of $n, r_{n}$ is obtained by fitting the data for $R_{n}(L)$ to $\simeq a L+r_{n}+b L^{-1}+c L^{-2}$ using four system size : $L, L-2, L-4$ and $L-6$, as indicated.

\begin{tabular}{|c|c|c|ccc|}
\hline & & & & & \\
$L$ & $R_{2}(L)$ & $a_{2}$ & $r_{2}^{(1)} / \ln 2$ & $r_{2}^{(4)} / \ln 2$ & $r_{2}^{(5)} / \ln 2$ \\
\hline 20 & 4.95205232373074 & 0.2138075040 & 0.9989748222 & 0.9999928713 & 0.9999877126 \\
28 & 6.66741530818944 & 0.2138074244 & 0.9996525352 & 0.9999971726 & 0.9999989449 \\
36 & 8.38060934985332 & 0.2138074200 & 0.9998432968 & 0.9999991645 & 0.9999998996 \\
44 & 10.0928119559937 & 0.2138074203 & 0.9999165184 & 0.9999996643 & 0.9999997718 \\
\hline
\end{tabular}

TABLE II. Rényi entropy $R_{2}(L, \mu=1)$ of the critical Ising chain in transverse field as a function of the system size $L$. The subleading constant $r_{2}\left(\mu_{c}\right)$ is extracted using three different fits (same as in Table $\mathbb{\Psi}$ ): $r_{2}^{(1)}$ is obtained by a fit to $R_{2}(L) \simeq$ $a L+r_{1}+b L^{-1}$ using the three following system sizes: $L, L-2, L-4 . r_{2}^{(4)}$ is obtained by a fit to $R_{2}(L) \simeq a L+r_{1}+b L^{-1}+\cdots e L^{-4}$ using the six system sizes $L, L-2, \cdots, L-10 . r_{2}^{(5)}$ is obtained by a fit to $R_{2}(L) \simeq a L+r_{1}+b L^{-1}+\cdots f L^{-5}$ using the seven system sizes $L, L-2, \cdots, L-12$. $a$ is the coefficient of the extensive (and non-universal) term, extracted from the seven-point fit above. These data show that $r_{1}$ converges to $\ln 2$ (limit $L \rightarrow \infty$ ). Similar results, with an even faster convergence, show that $r_{n \geq 2}\left(\mu_{c}\right)=\ln (2)$. With the present systems sizes and the present machine accuracy, adding more terms in the $1 / \mathrm{L}$ expansion does not increase the accuracy on $r_{2}$.

is particularly simple

$$
\langle G| \text { free }\rangle=\prod_{j=0}^{L / 2-1} \cos \frac{(2 j+1) \pi}{4 L},
$$

and leads to the following exact expression of the $n=\frac{1}{2}$ -
Rényi entropy:

$$
R_{\frac{1}{2}}(L, \mu=1)=L \ln 2+2 \sum_{j=0}^{L / 2-1} \ln \cos \frac{(2 j+1) \pi}{4 L} .
$$

Finally, an Euler-Maclaurin expansion gives the desired 
finite-size scaling, with a vanishing constant $r_{\frac{1}{2}}$ :

$$
\begin{aligned}
R_{\frac{1}{2}}(L, \mu=1) & =a_{\frac{1}{2}} L+r_{\frac{1}{2}}+o(1) \\
a_{\frac{1}{2}} & =\frac{2 K}{\pi} \\
r_{\frac{1}{2}} & =0
\end{aligned}
$$

where $K \simeq 0.91596559$ is Catalan's constant.

The constant term in $-\ln \langle G|$ free $\rangle$ has already been studied in Ref. 5. The situation where $|G\rangle$ is the groundstate of an antiferromagnetic spin- $\frac{1}{2}$ XXZ chain has also been considered.514 Such a scalar product is closely related to the notion of quantum fidelity.14 In terms of a classical $2 \mathrm{~d}$ Ising model, $-T \ln \langle G|$ free $\rangle$ is the boundary contribution to the free energy of a semi-infinite Ising model with free boundary conditions imposed at the edge. At the critical point, this is a well understood quantity from boundary CFT, and the subleading constant $r_{\frac{1}{2}}$ corresponds to $-2 \ln g$, where $g$ is the "groundstate degeneracy" discussed by Affleck and Ludwig. the present case of the Ising model, $r_{1}=0$ is in agreement with $g_{\text {free }}=1$. 0 This result has also been checked numerically in Ref. 22

\section{Critical behavior in the vicinity of $n=1$}

The results concerning the subleading constant $r_{n}(\mu=$ 1) are summarized in Fig. 5. The behavior of $r_{n}(\mu=1)$ has some similarity with that of $r_{1}(\mu)$ : the curves interpolates between 0 and $\ln 2$ with a slope at $n=1$ (resp. $\mu=1$ ) which increases as a function of the system size. Here again, it appears that the data for different values of $n$ and $L$ collapse onto a single curve when plotted as a function of $(n-1) L^{0.25}$ (inset of Fig. 5). The error bar on the exponent 0.25 are unfortunately large and difficult to estimate, but it indicates (a rather slow) divergence of the slope $\partial r_{n} /\left.\partial n\right|_{n=1}$ when $L$ increases. $r_{n}$ has also been computed for the classical Ising models on the square and triangular lattices, as in Sec. II A. The inset of Fig. 5 shows that the $r_{n}$ obtained from the corresponding transfer matrix calculations are in good agreement with those calculated from the ground state of the ICTF. This is a strong indication that, in a scaling region around $n=1$, $r_{n}$ defines a universal curve. The analogy between the effects of $\mu$ and $n$ suggests that $n-1$ is a "relevant" perturbation: going slightly below (resp. above) $n=1$ induces a drastic change in $r_{n}(\mu=1)$, which immediately (when $L=\infty)$ goes to 0 (resp. ln 2), as in high (resp. low) temperature phase of the $2 \mathrm{~d}$ Ising model.

\section{Vicinity of $n=\frac{1}{2}$}

The value $n=\frac{1}{2}$ can be treated exactly, as explained in Sec. IVB. However, the free-fermion calculation does not extend away from $n=\frac{1}{2}$. Still, at $n=0.5$ we observe (numerically) a crossing of the curves corresponding to

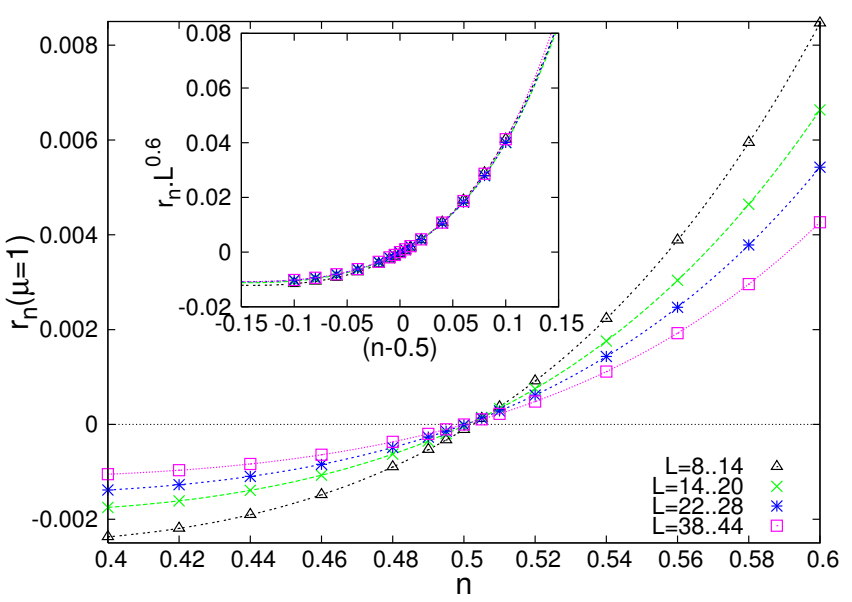

FIG. 6. (color online) Subleading constant $r_{n}$ of the Rényi entropy of the Ising chain (at the critical point $\mu=1$ ), in the vicinity of $n=0.5$. Inset : subleading constant multiplied by $L^{0.6}$ as a function of $(n-0.5)$.

different values of $L$ (see Fig. 6). This phenomenon, also observed at $n=1$, is reminiscent of a critical behavior, where the deviation away from $n=1 / 2$ would play the role of an irrelevant perturbation away from a fixed point. The data can also be collapsed onto a single curve, when the $\mathrm{y}$-axis is multiplied by a factor $\approx L^{0.6}$. However, contrary to the case $n=1$, this result indicates a reasonably fast convergence towards $r_{n}=0$ in the vicinity of $n=1 / 2$ (see the inset of Fig. 6).

\section{DISCUSSION AND CONCLUSIONS}

In the present Ising models, $r_{n}(\mu)$ seems to take only three discrete values. For example in the critical case, we find :

$$
r_{n}(\mu=1)=\left\{\begin{array}{cl}
0 & , n<1 \\
0.2543925(5) & , n=1 \\
\ln 2 & , n>1
\end{array}\right.
$$

This is quite different from other models described in terms of a free field compactified (with radius $R$ ) in the long-distance limit. In that case, which is better understood from a field theory point of view, the system describes a line of fixed points and the subleading copstant $r_{n}(R)$ continuously varies along that critical line 29

$$
r_{n}(R)=\ln R-\frac{\ln n}{2(n-1)} .
$$

We have discussed how the special values $n=\frac{1}{2}$, and $n=\infty$ are related to the $g$ factors associated to free and fixed boundary conditions of the Ising model. But so far, we do not know how to understand $r_{n=1}$ for the critical Ising model using CFT. This is certainly an interesting 
question for future studies. This is all the more challenging, as it seems (Secs. IV A and IV C) that replica methods for the Rényi parameter $n$ are not applicable to the Ising critical point for this quantity.

It is tempting to conjecture that crossings for $r_{n}(L)$ are observed whenever the underlying probabilities, $\sim p_{i}(\mu)^{n}$ describe a conformally invariant setup. It is indeed the case at $n=1 / 2$ (Ising boundary with free boundary conditions), but it is also realized for $n=1$, since it correspond to the bulk probabilities. It would be interesting to check this idea on other models, and to investigate the likely connection with the thery of line defects in conformally invariant systems.2021.

Acknowledgments - We wish to thank J. Dubail, S. Furukawa, A. Laüchli, Ph. Lecheminant, M. Oshikawa and H. Saleur for several useful discussions and suggestions.

The numerical calculations were done on the machine titane at the "Centre de calcul centralisé du CEA" under the project number p575.

\section{Appendix A: Probability of a spin configuration}

We consider an Ising chain in transverse field

$$
\mathcal{H}=-\mu \sum_{j=0}^{L-1} \sigma_{j}^{x} \sigma_{j+1}^{x}-\sum_{j=0}^{L-1} \sigma_{j}^{z}
$$

We assume $L$ to be even, as well as periodic boundary conditions $\sigma_{L}^{x}=\sigma_{0}^{x}$. We wish to find the ground state $|G\rangle$ of this Hamiltonian, and to compute all of his components in the basis of the eigenstates of the $\sigma_{j}^{z}$.

\section{Diagonalization}

As is well known, $\mathcal{H}$ can be expressed in terms of free fermions, using the Jordan-Wigner transformation :

$$
\begin{aligned}
\sigma_{j}^{x}+i \sigma_{j}^{y} & =2 c_{j}^{\dagger} \exp \left(i \pi \sum_{l=0}^{j-1} c_{l}^{\dagger} c_{l}\right) \\
\sigma_{j}^{z} & =2 c_{j}^{\dagger} c_{j}-1
\end{aligned}
$$

where the $c, c^{\dagger}$ satisfy the canonical anticommutation relations $\left\{c_{j}, c_{\ell}^{\dagger}\right\}=\delta_{j \ell}$. This allows to write the Hamiltonian as a quadratic form

$$
\mathcal{H}=-\sum_{j=0}^{L-1}\left(2 c_{j}^{\dagger} c_{j}-1\right)-\mu \sum_{j=0}^{L-1}\left(c_{j}^{\dagger}-c_{j}\right)\left(c_{j+1}^{\dagger}+c_{j+1}\right),
$$

where the fermions are subject to the following boundary condition :

$$
c_{L}^{\dagger}=-\exp (i \pi \mathcal{N}) c_{0}^{\dagger} \quad, \quad \mathcal{N}=\sum_{l=0}^{L-1} c_{l}^{\dagger} c_{l} .
$$

The parity operator $\mathcal{P}$ commutes with $\mathcal{H}$

$$
\mathcal{P}=\prod_{j=0}^{L-1} \sigma_{j}^{z}=\exp (i \pi \mathcal{N})= \pm 1,
$$

and because of Perron-Frobenius theorem, the groundstate lies in the sector $\mathcal{P}=+1$. Therefore, fermions are subjected to antiperiodic boundary conditions $c_{L}^{\dagger}=-c_{0}^{\dagger}$. $\mathcal{H}$ can finally be diagonalized by a Bogoliubov transformation :

$$
\begin{gathered}
c_{j}^{\dagger}=\frac{1}{\sqrt{L}} \sum_{k} e^{i k j}\left(\cos \theta_{k} d_{k}-i \sin \theta_{k} d_{-k}^{\dagger}\right) \\
k \in\{(2 l+1) \pi / L \mid-L / 2 \leq l \leq L / 2-1\} \\
\sin 2 \theta_{k}=\frac{\mu \sin k}{\sqrt{1+2 \mu \cos k+\mu^{2}}} \\
\cos 2 \theta_{k}=\frac{1+\mu \cos k}{\sqrt{1+2 \mu \cos k+\mu^{2}}}
\end{gathered}
$$

The new fermions operators $d_{k}, d_{k}^{\dagger}$ satisfy the necessary anticommutation relations, and diagonalize $\mathcal{H}$ :

$$
\begin{aligned}
\mathcal{H} & =\sum_{k} \varepsilon_{k}\left(d_{k}^{\dagger} d_{k}-1 / 2\right) \\
\varepsilon_{k} & =2 \sqrt{1+2 \mu \cos k+\mu^{2}} .
\end{aligned}
$$

$\varepsilon_{k}>0$ ensures that the ground-state $|G\rangle$ is the vacuum $|0\rangle$ of the $d_{k}$.

\section{Exact formulae for the spin probabilities}

We define $P_{j}^{\sigma}$ as the projector onto the $|\sigma= \pm 1\rangle_{j}^{z}$ state:

$$
P_{j}^{+}=c_{j}^{\dagger} c_{j} \quad, \quad P_{j}^{-}=c_{j} c_{j}^{\dagger} .
$$

$p_{i}$ is then given by

$$
p_{i}=p\left(\sigma_{0}, \ldots, \sigma_{L-1}\right)=\left\langle 0\left|P_{0}^{\sigma} P_{1}^{\sigma} \ldots P_{L-1}^{\sigma}\right| 0\right\rangle .
$$

Using Wick's theorem, this correlator reduces to a Pfaffian. To compute it, we need to calculate the four types of contractions $\left\langle c_{j}^{\dagger} c_{\ell}\right\rangle,\left\langle c_{j} c_{\ell}^{\dagger}\right\rangle,\left\langle c_{j}^{\dagger} c_{\ell}^{\dagger}\right\rangle,\left\langle c_{j} c_{\ell}\right\rangle$, which can be done using Eq. (A7). It is worth noticing that all these correlators are real in this particular model. We write a generic projector as :

$$
P_{j}^{\sigma}=f_{j}^{\dagger} f_{j}
$$

with $f_{j}^{\dagger}=c_{j}^{\dagger}$ for $\sigma=+1$ and $f_{j}^{\dagger}=c_{j}$ for $\sigma=-1$. Then :

$$
\begin{aligned}
p_{i}^{2} & =\left\langle f_{0}^{\dagger} f_{0} f_{1}^{\dagger} f_{1} \ldots \ldots f_{L-1}^{\dagger} f_{L-1}\right\rangle^{2} \\
& =\left\langle f_{0}^{\dagger} f_{1}^{\dagger} \ldots f_{L-1}^{\dagger} f_{0} f_{1} \ldots f_{L-1}\right\rangle^{2} \\
& =\operatorname{Pf}^{2}\left(\begin{array}{cc}
A & B \\
-B & -A
\end{array}\right)
\end{aligned}
$$


where $A$ is antisymmetric, $B$ is symmetric, and Pf denotes the Pfaffian. The matrix elements of $A$ and $B$ are

$$
\begin{aligned}
& A_{j \ell}=\left\langle f_{j}^{\dagger} f_{\ell}^{\dagger}\right\rangle \quad, \quad \ell \geq j \\
& B_{j \ell}=\left\langle f_{j}^{\dagger} f_{\ell}\right\rangle
\end{aligned}
$$

Using the relation $\mathrm{Pf}^{2}=$ det, Eq. (A18) simplifies into

$$
\begin{aligned}
p_{i}^{2} & =\operatorname{det}\left(\begin{array}{cc}
A & B \\
-B & -A
\end{array}\right) \\
& =\operatorname{det}\left(\begin{array}{cc}
A+B & B \\
0 & B-A
\end{array}\right) .
\end{aligned}
$$

Eq. A22 follows from Eq. (A21) by adding the second column to the first, and then the first row to the second.
Finally,

$$
p_{i}=\operatorname{det}(A+B)=\operatorname{det} M,
$$

where $M$ is a $L \times L$ matrix with elements

$$
\begin{aligned}
M_{j \ell} & =\left\langle f_{j}^{\dagger}\left(f_{\ell}^{\dagger}+f_{\ell}\right)\right\rangle \\
& =\frac{1}{2} \delta_{j \ell}+\frac{\sigma_{j}^{z}}{2 L} \sum_{k} \cos \left[k(j-\ell)+2 \theta_{k}\right]
\end{aligned}
$$

At the critical point $(\mu=1), \theta_{k}=k / 4$ and the matrix elements simplify even further :

$$
M_{j \ell}=\frac{1}{2} \delta_{j \ell}+\frac{(-1)^{j-\ell} \sigma_{j}^{z}}{2 L \sin [\pi(j-\ell+1 / 2) / L]} .
$$

1 L. Amico, R. Fazio, A. Osterloh, and V. Vedral, Rev. Mod. Phys.80, 517 (2008).

2 S. Furukawa and G. Misguich, Phys. Rev. B75, 214407 (2007).

3 D. S. Rokhsar and S. A. Kivelson, Phys. Rev. Lett.61, 2376 (1988)

4 C. L. Henley, J. Phys.: Condens. Matter 16, S891 (2004).

5 J.-M. Stéphan, S. Furukawa, G. Misguich and V. Pasquier, Phys. Rev. B80, 184421 (2009).

${ }^{6}$ E. Fradkin and J. E. Moore, Phys. Rev. Lett.97, 050404 (2006).

7 B. Hsu, M. Mulligan, E. Fradkin and Eun-Ah Kim, Phys. Rev. B79, 115421 (2009).

8 I. Affleck and A. W. W. Ludwig, Phys. Rev. Lett.67, 161 (1991).

9 J. L. Cardy, Nucl. Phys. B 324, 581 (1989).

10 L. Onsager, Phys Rev. 65, 117 (1944).

11 R. Houtappel, Physica 16, 425 (1950).

12 H. A. Kramers and G.H. Wannier, Phys Rev. 60, 252 (1941)

13 J. Sawada, SIAM J. Comput. 31, 259 (2001).

14 L. Campos Venuti, H. Saleur, and P. Zanardi, Phys. Rev. B79, p92405 (2009).

15 R.J. Baxter, Exactly Solved Models in Statistical Mechanics (Dover Publication, Mineola, 1982).

16 S. Katsura, Phys Rev. 127, 1508 (1962).

17 M. Levin and X.-G. Wen, Phys. Rev. Lett.96, 110405 (2006).

18 A. Kitaev and J. Preskill, Phys. Rev. Lett.96, 110404 (2006).

19 M.A. Metlitski, C.A. Fuertes, and S. Sachdev, preprint arXiv:0904.4477.

20 V. B. Petkova and J.-B. Zuber, Phys. Lett. B 504, 157 (2001).
${ }^{21}$ M. Oshikawa and I. Affleck, Phys. Rev. Lett.77, 2604 (1996)

22 J. Dubail, J.L. Jacobsen and H. Saleur, Nucl. Phys. B 834, 399 (2010).

23 This quantum/classical correspondence works in a rather straightfoward way for simple constrained models (such as dimer models or vertex models). For other models, such as the Ising model considered in this paper, some additional care is needed to define the geometry of the $A / B$ boundary at the microscopic level. In the particular case of $2 \mathrm{~d}$ classical Ising models, the spins living at the frontier between $A$ and $B$ have to be "duplicated" to insure that the decomposition induced by the classical spin configurations is indeefha proper Schmidt decompostion of the RK state. See Ref 5 for more details.

${ }^{24}$ In the quantum point of view, where one studies the entanglement in a RK wave-function, the dominant $(\sim L)$ contribution is the boundary (alsorcalled "area") law.

25 The field-theory prediction of Ref is $r_{1}\left(T=T_{c}\right)=\ln (2)$ and does not agree with our numerical calculations Remark: $r_{1}$ corresponds to $S_{0}^{(x)}$ in the notatienfof Ref.

${ }^{26} T_{c}=2 / \ln (1+\sqrt{2})$ on the squate lattice 10 .2 and $T_{c}=$ $4 / \ln (3)$ on the triangular lattice 11

27 We also use the property that, for periodic boundary conditions, $\prod_{i} \sigma_{i}^{z}=1$ in the ground state $|G\rangle$. Since we work in the $\sigma^{z}$ basis, this reduces by another factor two the number of probabilities to compute.

28 In fact, the analysis of Sec. IVd suggests that $r_{n}\left(\mu_{c}\right)$ flows to its ferromagnetic boundary condition limit, $\ln (2)$, as soon as $n>1$.

29 See Eq. (77) in Ref. 5 . The following normalization of the radius is used: $R=1$ for free fermions, and $R=\sqrt{2}$ at $S U(2)$ symmetric (self-dual) point of the XXZ chain. This corresponds to a lagrangian $\mathcal{L}=\frac{1}{8 \pi}\left(\partial_{\mu} \varphi\right)^{2}$, where the field is compactified on a circle of radius $R: \varphi=\varphi+2 \pi R$. 